\title{
Framework to Practical Introductory Classes about Development of Information System
}

\author{
Ayrton Saito, Raphael Garcia, Mauricio Araujo Dias, Miriam Maria Pedrosa and Andreia Cristiane Silva Wiezzel \\ UNESP_Univ. Estadual Paulista, Roberto Simonsen, 305 - Presidente Prudente Sao Paulo 19060 900, Brazil
}

\begin{abstract}
Many information system students have difficulty to learn how they can develop information systems using tools which already exist. For this reason, the goal of this paper is present a tool which was created to support teachers to conduct introductory practical classes for students who begin to learn how to develop system. For this propose, this work describes a framework that helps these students during their introductory practical learning of information system. This framework follows part of MVC model and uses the PHP programming language. The results presented in this paper show that the framework assists teachers performing introductory practical classes for beginner students. This work aims to contribute for students understand the development of system more quickly and easily.
\end{abstract}

Key words: Framework, practical classes, teaching, information system.

\section{Introduction ${ }^{1}$}

Due to the Information Age, the digital IS (information systems) used to organize information are becoming more and more evident on people and business's daily lives. Due to a daily increasing of data production the IS gained relative importance on labour market, consequently the intensification of development of IS's become a necessity. In parallel, an insufficient supply of skilled labour for development of IS's has caused pay increase and a rise in the number of job vacancies offered for these professionals. The benefits provided for developers of IS's on the labour market have stimulated students to look for graduation courses in the area of information systems. However many of this students have difficulty in learn to develop IS's from the use of development tools which already exist. Thus, the creation of new tools to teach introductory practical classes about IS's development for this students become fundamental in courses of information system.

Corresponding author: Mauricio Araujo Dias, Ph.D., professor, research fields: hardware, FPGA, elliptic curves, digital image processing.
Among the tools used for professionals to develop IS's as well to teach students to develop IS's, the most promising has been the framework. That's because a framework is a tool to development of IS's focused on: solving problems, rising in productivity and reusing of code. The framework of this work, for instance, generate IS's in an intuitive way, reusing code already developed. The IS developed in this framework can use standard functionalities such as insertion, alteration, removal, relation and data search, besides other actions which can be customized by developers. This tool provides, with simplicity, the development of IS's, contributing to practical learning of students in courses of information systems.

Just for having an idea of the potential offered by a framework for development of IS's, we find on literature a comparison between development of application with and without the use of framework [1]. The results indicated that for any project, big or small, the use of a framework increases the production capacity. However, in relation to the frameworks of existing on the market, although they are full and robust, usually require previous knowledge in IS's development. In addition, they usually present 
inaccessible code and they are extensive which limit its use as tool to teach student developing IS's.

Some models of tools can be used as base to creation of news frameworks, for example, the MVC (model view controller) model which was used in Refs. [2, 3]. The MVC allows the development of a very well organized and modular structure. However, when it is used in full, the understanding and the learning of the students, which do not have so much familiarity with the model, can be damaged. Thus, the MVC is an important tool to serve as a basis to creation of frameworks, however this model must be used together with another models of tools to become more appropriate to teach the development of IS's.

In addition, an appropriate tool to teach development of IS's also must to offer methods and functionalities which allow the students to create complex actions, but they also have to be easily comprehended and debugged. For this reason, the tools based in paradigms as the POA [4], aspect oriented programming, using to control the access and register of the log, are inappropriate to teach IS's development, since, due its structure, this paradigm makes the depuration of the source code very difficult.

Others tools are present in Refs. [5, 6], however these papers present systems dedicated to solve specific problems of market and e-commerce, respectively. In this way, although the positive results described in both, the cited papers do not present appropriate tools to teach students to develop another type of IS's besides to the proposed areas.

In other words, none of the previously cited articles present a tool adequate enough to help beginners students in information system courses to understand, in practice, how to develop IS's easily, clearly and objectively. Therefore, these students still depend on the creation of a tool more appropriate to help them in their early stage of learn.

In face of this reality, the goal of this paper is present a tool created to help teachers giving initial practical classes for beginners students who will learn to develop IS's. For this propose, this paper describes a framework used as tool to support beginners students during their practical introductory learning of Information System. This framework follows, partially, the MVC model, using PHP programming language [2, 3]. The use of PHP language matched with other technologies allows the creation of an ideal framework for the rapid development of IS's. This tool allows to build systems that are: complete, accessible via internet, and independent of platforms, as its purpose.

To simplify the understanding of this work, we organized this paper as follows: The Section 2 presents the theoretical fundamentals; Section 3 comments material and methods; The results obtained with the use of the proposed tool are presented and discussed in Section 4. The Section 5 presents the conclusion.

\section{Theoretical Fundamentals}

A framework is an environment that allows the development of projects even faster and easier, from source code, libraries, classes, functions and methodologies [7]. The characteristics of a framework:

- Reuse: to enable the use of available codes and modules. The documentation of the available codes and modules must be detailed and easy to understanding;

- Extensibility: should allow the inclusion of libraries and tools which add new features that there are not in original framework;

- Security: a framework cannot be fragile in relation to their use. By using the framework, the user cannot lead it to a state incapable of carrying out their basic functionalities, as well cannot to make it unsafe and inconsistent, in any action performed, independently, of the profile who manipulates;

- Efficiency: it must to consider response time and security, besides to ensuring the consistency and integrity of data;

- Coverage: a framework should be able to solve 
the higher number of problems, this way, providing useful systems for many situations.

The framework presented in this paper addresses the CRUD (create, read, update and delete) actions and develops IS's using JavaScript, PHP, HTML, CSS and XML [8-10].

Information system is an expression used to describe a system which includes, among other things, methods to obtain, to process, to transmit and to distribute data that are the representation of some information [5].

\section{Materials and Methods}

The framework presented in this paper provides IS's for manipulation, organization and data relationship. The framework focuses on the development of the back-end of IS's quickly and effectively. This framework accelerates the development of systems since it reuses already existing codes. The main functionalities of the framework are: generation of the back-end of systems for inclusion, change, removal, research and data relationship; reuse of available codes; delivering of Ajax methods; possibility of customization, inclusion forms and change of data; definition of field masks; definition of hierarchy between tables from database; provision of methods for inclusion of functionalities for the back-end. Besides the cited functionalities, the framework is supported by a collection of libraries which the student can use and a system with resources to help in the development and the rise in productivity.

The standard interface of the framework, as shown in Fig. 1, was developed using HTML and CSS for rendering and stylization of the components. The JavaScript was included to improve the feedback and the interaction with the user. Despite of the framework to provide a standard interface, any user can modify and customize the interface, but the user must preserve the components that are useful for rendering grids, forms and other components.

The framework has some essential settings which determine some aspects about the production environment. The configurations are stored in a simple file .php located in config directory that defines a vector inside a global variable. To change the configurations, just carry out the modifications directly on the source code or with the assistance of the control and operation system. The control and operation system is another resource that the framework

\section{Basic structure for interfaces}

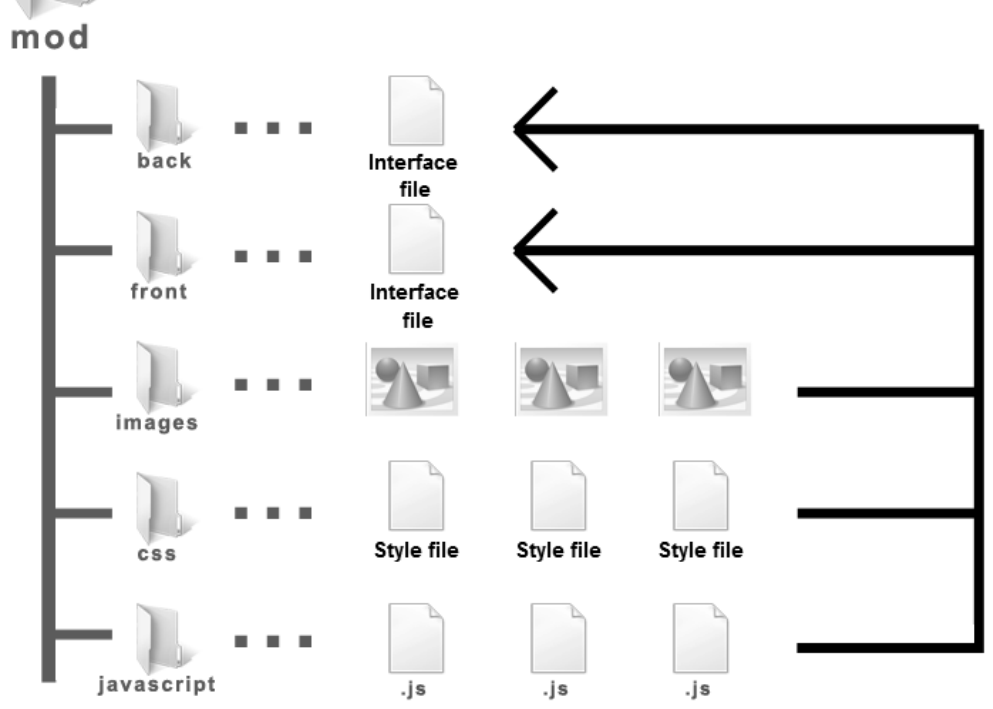

Fig. 1 Organization of components of the interface. 
offers to increase the productivity and to abstract some actions for the students. This control and operation system of the framework is an environment that helps students to carry out actions through an interface from the framework. The main actions available are: change of configuration file; execution of a SQL script in the current database; edition of files from modules; installation and uninstall of current modules; creation of new projects; automatic generation of modules.

The configurations file has several predefined values which can and should be modified to define questions about each project. This way, configurations become more flexible towards the needs of each environment. The basic settings that should be defined are: the name of the theme in question; the settings of connection of the standard database; the system directory for upload; the base directory where the framework is located; the standard protocol of internet to be used; the codification of characters which must be considered.

To provide greater security for the system and in order to allow the use of the chosen structure of directories, it was decided that the URLs (Uniform Resource Locator) must be processed instead of being used in the usual form. In addition, on the electronic pages addresses, we can always notice the permanent presence of files extensions, normally at its end, what allows to recognize the use of particular technology in that page. Some examples of extensions present in these pages are: .php, .html, .asp, .aspx, etc.

Furthermore, for dynamic pages, as PHP and ASP, we can notice the presence of some parameters, in other words, values that are used for some finality, as: change of content of the page, identify a particular page, information for password recovery, among others. Although the use of parameters is required at addresses of electronic pages, often this shows us the vulnerabilities of a certain page or system. It can do people with malicious intent to use resources to damage or undue access.

For this reason, to hide the parameters and the extension of developed pages in the framework in order to improve the safety and also became the URLs aesthetically pleasing, a variety of rules was defined by Htaccess (hypertext access). The Htaccess is defined in a file .htaccess and allows the change of permissions, access and, in the case of URLs, the manipulation of rules, for its processing and adequacy. Regarding the configuration, it is necessary only to define the topic in question. Based on that, the rules of Htaccess have full autonomy to process the URLs from production environment. In addition to the contribution of the Htaccess, there is a pre-processing of the URL using the file index.php which will be responsible to do the access of file from the defined URL.

This framework was organized in modules developed with specific purposes. A module is a small portion of files that are structured in directories, which follow the rules of the framework. This means that it is possible to develop and implement modules according to each project or need. The framework has essential modules which should be part of all projects. It is necessary for the IS to provide standard functionalities to the user.

The modules have the follows characteristics: they are developed only once and can be reused to any project; they are generated in a fast way, through resources offered by the framework; they are extensible, i.e., it is possible to modify it to add new functionalities; they can be overwritten (the structure of directories of the framework allows a module to be overwritten for any project, without change the original codification); they are dependent, i.e., a module can define a dependence, if it needs some information from another module; they are well organized; a module must be small and have few functionalities to be reused.

Modules are organized in directories. The structure of directories of the framework was elaborated to separate different types of files and to allow the overlay of directories. The basic structure of 
directories is represented in Fig. 2.

One of the most important directories is the directory of modules (mod). The mod has the standard modules for any project. It has the modules already developed following the proposed rules by the framework, as shown in Fig. 3.

Besides the organization of directories, the structure of internal files of the framework represents an
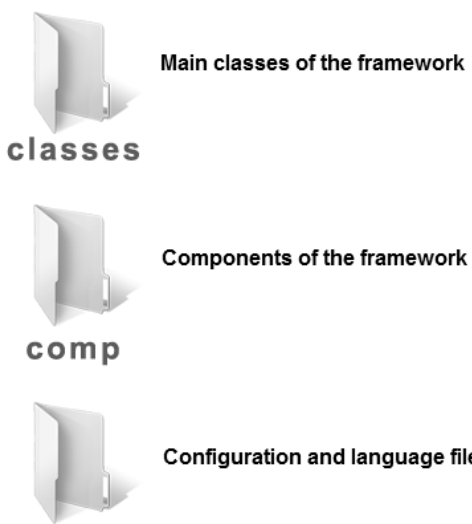

Configuration and language files config

Resources and library files

lib abstraction of the MVC model. In other words, the framework does not use the model according to the rules. The purpose of abstraction is to facilitate the learning and the understanding of the structure of the files. This way, using the object orientation and the abstraction of the MVC model to the rendering of an electronic page, the content is separated, usually, in three files:

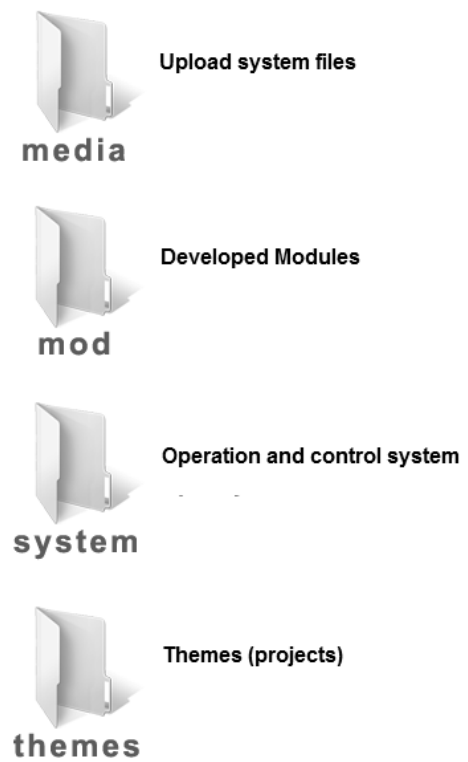

Fig. 2 Basic structure of directories of the framework.

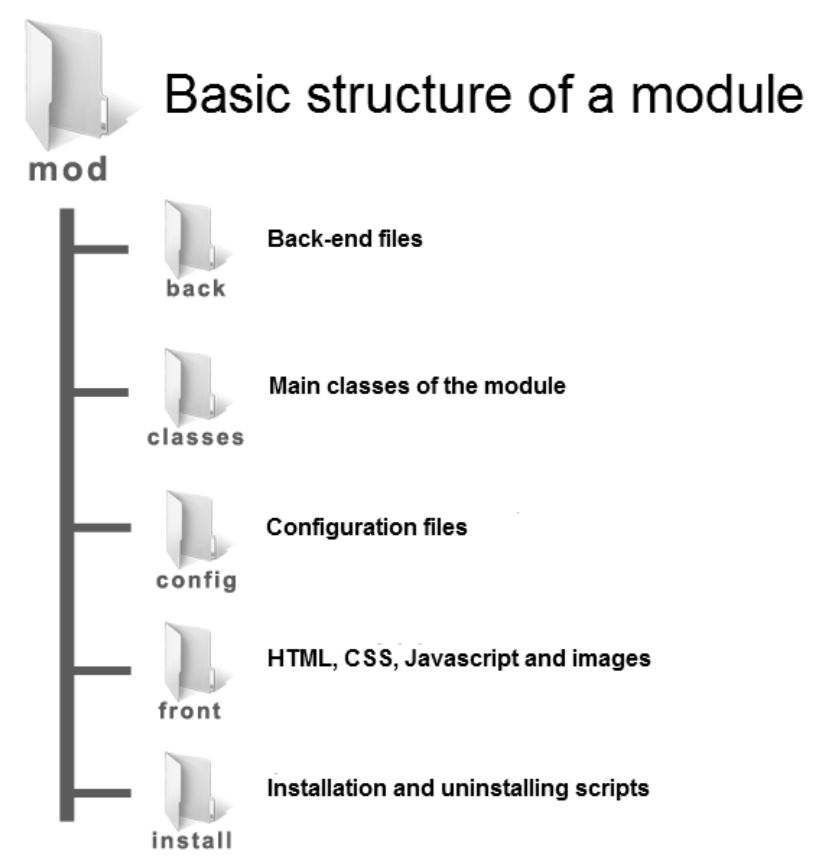

Fig. 3 Main directory of the framework. 
Class: following the paradigms of object orientation, the essential information of a module are detained, serving as the basis to obtain information;

DAO class (data access object): holding the methods to consult the database;

Component: obtains information from "Class" and performs the rendering of form, grids, tables, among other structures.

It is important to point out that the well organized structure of directories and files is fundamental for the framework to fulfill its purpose related to be extensible with the intention to attend the needs of several projects.

The framework has several classes created to facilitate the development of pages and new functionalities to the framework. The greater difficulty was to codify a structure which could identify, through rules, how to behave and to carry on these tasks in a generic way. However, by using the object orientation and based on the most common possibilities for the registration of data, the rules were properly implemented in order to serve as solution to larger amounts of possible problems.

To generate a module, firstly, the framework creates the scripts of tables of the databases, which are related to the module to be generated, as shown in Fig. 4. Then, by passing parameters, the framework identifies the mandatory information and performs the construction of the CRUD. However, some small adjustments must be made in the classes of the newly created modules. It is worth pointing out that the automation tool of the framework generates all essential files and directories that would be, usually, manually performed. This way, it has a significant gain of time in the phase of development of modules.

By executing the script presented in Fig. 4, the framework creates a table named "cr_curso". As a suggestion to standardize the nomenclature, tables must have a nominal reference to the identification of the class/module in the PHP program, i.e., they must have similar names. Thus the "cr" prefix is only an adopted rule to identify the classes and the tables of a given module, but the "cr" prefix is optional.

After the execution of the generator module, the following files are created: create.phtml, index.phtml, update.phtml, relate.phtml and view.phtml. They are created to allow the rendering of pages from CRUD. Fig. 5 illustrates an example of code found in the file create.phtml, which is used to render a page of the back-end.

As all cited files are similar, we are going to comment only the create.phtml file which is responsible to display the form for insertion of curses. In line 2, we have the inclusion of class CrCurso. In line 3 , we have the definition of global variable “\$models”. In line 4, in the case of variable \$models is empty, it receives an instance from class CrCurso. Finally, in line 5, the "render" method is called to perform

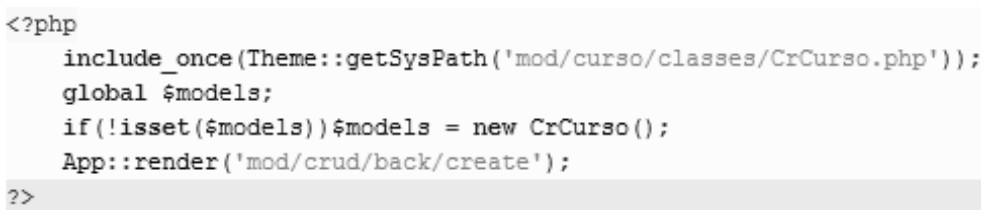

Fig. 4 Definition of the fields of table "cr_curso".

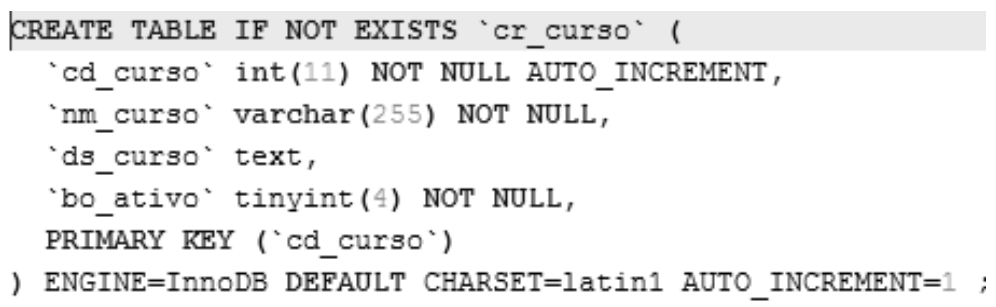

Fig. 5 Content of the create.phtml file. 
the rendering of the page which will contain the form for insertion of curses. To reuse a model in other project, it is convenient that the installation and uninstalling files are available for carrying out the implementation of the module. This way, for carrying out the installation, two files with extension ".sql” must be created. They must contain the scripts for creation and deletion of tables of the database. The files must be nominated as "install.sql" and "uninstall.sql", respectively. The script for creation of tables is the same showed in Fig. 4 and the script for deletion can be represented with the content "DROP TABLE name”.

\section{Results and Discussion}

In order to verify if the framework presented in this paper helps teachers in their introductory classes for beginners students which will learn to develop IS's and if the systems generated by the framework present characteristics that are similar to IS's developed in the labour market, we used the framework with a group of students and collected information regarding to different aspects related to the IS's development. The criteria used to verify the framework and its IS's properties were: performance of students learning to develop IS's with the use of the framework; facility of learning the structure and the functioning of the framework; the average time consumed to the development of ISs with the use of framework; capacity of IS's developed by the framework and the potential management of its ISs by users.

To evaluate the performance of students learning to develop ISs with the use of the framework, one group of ten students it was invited to participate of one introductory class about development of ISs with the use of framework. Following the teacher's orientation, the students learn to develop a basic IS in only thirty minutes. Subsequently, the same students showed to be able the development of other basic IS also in thirty minutes, however, this time, without teacher's orientation.

The assessment of the average time consumed to develop one IS by using the framework taken into account the development of one small IS for cadastre of clients with and without the use of the framework. The framework has several modules which can be installed in any project. Therefore, when using the project creation tool with the help of the control and operation system, the IS was developed in less than thirty minutes. However, if necessary the development of a new module, there are two possibilities: the manual creation, following the framework pattern and the creation with the assistance of the module generator. In the first case, by using database modeling, the creation is done automatically. In the second case, the manual development of a simple IS for cadastre of clients (without using the framework), considering all access controls and user permissions, it takes about twenty hours to be concluded.

Based on the number of students and the number of system access, we could observe the capacity of the system using a simple server of a commercial hosting. According to the users and the managers who used the IS, the access time and the time to answer were satisfactory, with less than one second of duration in all accesses. Therefore, we could confirm that despite of its generic form, an ordinary internet server can host, without overload, the ISs developed by the framework.

Considering the results obtained to the performance of students learning to develop IS's with the use of the framework, facility of learning the structure and the functioning of the framework, the average time consumed to the development of ISs with the use of framework, capacity of IS's developed by the framework and the potential management of its ISs by users, it was noted that the framework presented in this paper helps teachers in their practical introductory classes for beginners students which will learn to develop IS's and also it was noticed that the ISs, generated by the framework, show similar characteristics to the ISs developed in the labour market. 
Another important point is that the tool presented in this paper has accessible code, it is extensible and students do not require previous knowledge in development of ISs to work with, unlike frameworks which exist in the market. By use only partially the MVC model together with other models of tools, the framework presented in this paper shows a well organized and modular structure, that is easy to understand and, for that, more appropriated for teaching the development of ISs than the form which the model is used in Ref. [2] or in Ref. [3]. In addition, the tool of this paper offers methods and functionalities that allow the student to create complex actions, easily to be understood and debugged, in contrast to what we have found in Ref. [4]. Moreover, the framework allows to develop many types of ISs, as opposed to the ISs described by Ref. [5] or Ref. [6].

\section{Conclusion}

This paper showed a tool created to help teachers in their practical introductory classes for beginner students which will learn to develop ISs. It's a supporting tool to students who are beginning the learning process about the development of ISs in Information System courses.

We can mention the following contributions of this work: students' attainment to IS's development accessible via the internet, “multi-platform"; productivity in the IS's development; code's reusing; automatic generation of code; description of the rules structure and description of the framework definition. However, the most significant contribution provided by this work is to allow students of Information Systems understanding the development process of ISs, even without previous knowledge about the subject.

Therefore, we conclude that the framework presented by this paper is a tool that contributes to the early stage of learning for students who will learn to develop ISs in practical classes in Information Systems courses.

\section{References}

[1] Vuksanovic, I. P., and Sudarevic, B. 2011. "Use of Web Application Frameworks in the Development of Small Applications." In Proceedings of the 34th International Convention MIPRO, 458-62.

[2] Wang, G. 2011. "Application of Lightweight MVC-like Structure in PHP.” Presented at the 2011 International Conference on Business Management and Electronic Information (BMEI).

[3] Leff, A., and Rayfield, J. T. 2001. "Web-Application Development Using the Model/View/Controller Design Pattern.” Presented at the Fifth IEEE International Enterprise Distributed Object Computing Conference (EDOC '01). doi: 10.1109/EDOC.2001.950428.

[4] Hokamura, K., Naruse, R., Shiozuka, M., Ubayashi, N., Nakajima, S., and Iwai, A. 2009. "AOWP: Web-Specific AOP Framework for PHP.” Presented at the 24th IEEE/ACM International Conference on Automated Software Engineering (ASE'09). doi: 10.1109/ASE.2009.45.

[5] Guo, W. D. 2008. "Development of Web Information System of Corporation: An Exploring Research.” Presented at the International Symposium on Intelligent Information Technology Application Workshops (IITAW’08). doi: 10.1109/IITA.Workshops.2008.201.

[6] Andreoli, J. M., Pacull, F., and Pareschi, R. 1997. "XPECT: A Framework for Electronic Commerce." IEEE Internet Computing 1: 40-8. doi: 10.1109/4236.612214.

[7] Minetto, E. L. 2007. Frameworks Para Desenvolvimento Em PHP. Sao Paulo: Novatec.

[8] Korpela, J. 1998. "Lurching toward Babel: HTML, CSS and XML." Computer 31: 103-6. doi: 10.1109/2.689682.

[9] Simeon, J., and Wadler, P. 2003. "The Essence of XML.” In Proceedings of the 30th ACM SIGPLAN-SIGACT Symposium on Principles of Programming Languages (POPL'03), 15-7.

[10] Thomas, B. 1999. “Cascade Effects.” IEEE Internet Computing 3: 89-90.doi: 10.1109/4236.747329. 